\title{
Reviews of Research on Mechanical Fault Diagnosis in GIS
}

\author{
Yuqi $\mathrm{Cai}^{1, *}$, Liang $\mathrm{Jin}^{2}$ \\ ${ }^{1}$ College of Information Science and Engineering, Huaqiao University, 361021,Xiamen, China \\ ${ }^{2}$ College of Information Science and Engineering, Huaqiao University, 361021,Xiamen, China
}

\begin{abstract}
Gas Insulated Switchgear (GIS) has been widely used in recent years due to their advantages of small floor area, high reliability and good safety. It has arised a series of problems. At present, researchers at home and abroad have done more in-depth research on insulation fault, but research on mechanical fault is insufficient. Firstly, this paper reviews the research status of mechanical fault detection technology in GIS at home and abroad. Secondly, the aspect of the principles of mechanical fault in GIS, fault judgment and location technology, and early fault prediction and status evaluation in GIS were summarized. Finally, the paper prospected the future research direction on this basis.
\end{abstract}

\section{Introduction}

Gas Insulated Switchgear (GIS), also known as the SF6 fully enclosed combined apparatus, in addition to the transformer, it is all enclosed within a grounded metal shell which is filled with $0.34 \mathrm{MPa}$ to $0.4 \mathrm{MPa}$ SF6 gas as insulation and arcing medium, including circuit breaker, disconnecting switch, grounding switch, current transformer, voltage transformer, lightning arrester, busbar, connecting pipe and transition element[1]. Compared with conventional open type substation, it has many advantages such as small occupied area and space, high operation reliability, good safety, long maintenance period and little influence from external environment, so it is widely used in urban substation [2].

In recent years, the load of GIS equipment of various voltage levels in the power grid has been increasing rapidly, but the resulting problems can not be ignored. According to the statistics of national power reliability, with the continuous improvement of GIS availability coefficient, the forced outage rate has also increased significantly, indicating that the fault problem of GIS has increased to a certain extent [3]. Therefore, compared with conventional electrical equipment, GIS has some disadvantages in operation and maintenance. The main points are as follows: (1) Key components of the equipment are enclosed in the metal shell, and it is very difficult for maintenance personnel to find fault signs. (2) The internal space of GIS is small, but the structure is complex. So the connection between devices is close and the mutual influence is big. It is easy to produce chain reaction by this, increasing the difficulty of fault judgment and positioning. (3) Faults of GIS affects a large range, and it

\footnotetext{
*Corresponding author: cyq20200213@163.com
} 
takes a long time for check and maintenance, generally more than two weeks [4]. Therefore, more effective on-line monitoring and fault diagnosis techniques are adopted Points is necessary.

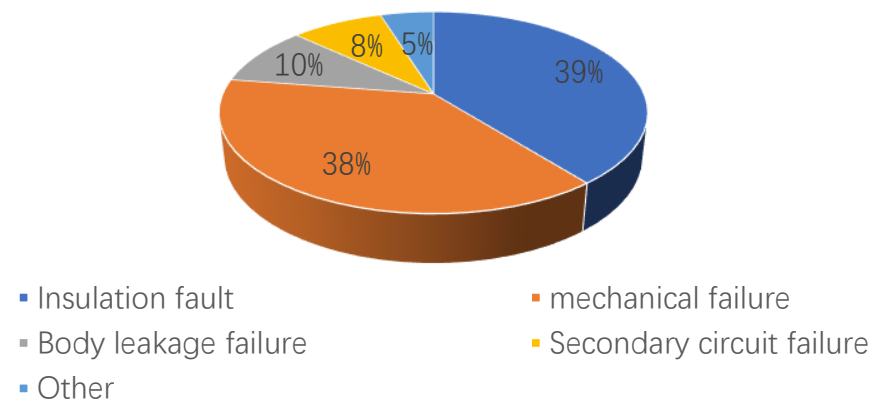

Fig. 1. Fault statistics of GIS

According to various statistical data, GIS faults can be roughly divided into mechanical faults, insulation faults, secondary circuit faults, body leakage faults, other faults, etc[3]. The proportion of each fault is shown in figure 1. As can be seen from figure 1, the main faults of GIS are insulation faults and mechanical faults. Since the birth of GIS in the 1960s, there have been many systematic and in-depth studies on its discharge fault, but the research on mechanical fault is insufficient [4]. Therefore, we need to further study its mechanism and methods of detection and diagnosis.

This paper systematically sorted out the research status of GIS mechanical fault diagnosis at home and abroad, and summarized the characteristics of GIS mechanical fault, fault judgment and positioning technology, GIS early fault prediction and reliability evaluation, and clarified the existing problems in fault detection and future development direction. The overview framework is shown in figure 2 .

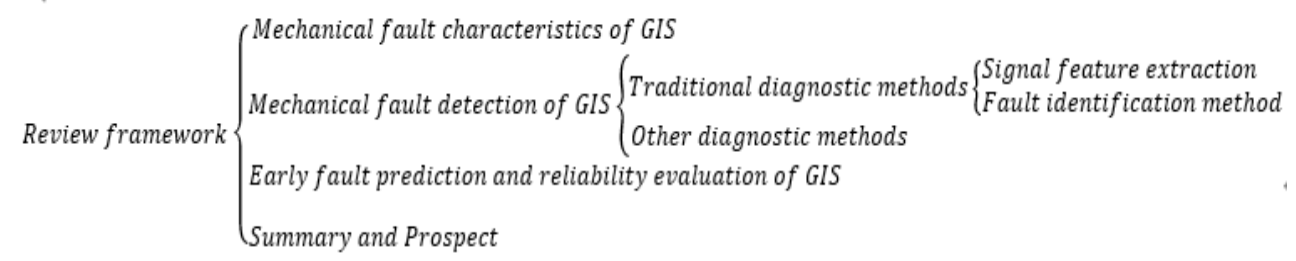

Fig.2. Review structure of Research on Mechanical Fault Diagnosis in GIS

\section{Mechanical failure characteristics of GIS}

To diagnose GIS mechanical fault, it is necessary to clarify GIS fault mechanism and its typical characteristics. There are three kinds of vibration caused by GIS mechanical failure, including electromagnetic force, electric force and ferromagnetic resonance, Vibration caused by switch operation, and vibration caused by mechanical failure of circuit breaker [6]. Circuit breaker, as an important GIS device, is most prone to mechanical failure. Secondly, dynamic and static contact system and the isolation switch are also common mechanical failure devices. Voltage transformers, electromagnetic contactors and current transformers all work by magnetic flux characteristics, and mechanical and electrical faults often interweave on them.

According to the different vibration frequencies, the GIS state can be distinguished. At present, most researches on GIS vibration frequency are conducted through finite element analysis software simulation or modal test $[7,8,9]$, and some are also conducted through 
experimental platform for simulation test analysis. The vibration frequencies of different causes obtained from the study are summarized in table 1 .

Table 1. Causes and Frequencies of Vibration in GIS.

\begin{tabular}{|c|c|}
\hline GIS vibration principle & Frequency characteristics of vibration signals \\
\hline Partial discharge & The main frequency of vibration is $5-30 \mathrm{kHz}$. \\
\hline $\begin{array}{c}\text { Conductive particles } \\
\text { Electromagnetic force, electromotive force, } \\
\text { electrostatic force }\end{array}$ & $\begin{array}{c}\text { It is mainly } 100 \mathrm{~Hz} \text {, and the vibration caused by } \\
\text { electric power is very weak when the high- } \\
\text { frequency signal is above } 300 \mathrm{~Hz} .\end{array}$ \\
\hline Contact surface current shrinkage & $\begin{array}{c}\text { The main frequency is } 100 \mathrm{~Hz} \text {, and the GIS high } \\
\text { voltage conductor has some defects such as poor } \\
\text { mechanical contact, which will lead to the } \\
\text { increase of vibration signal amplitude, and the } \\
\text { increase of signal component amplitude of } 200 \mathrm{~Hz} \\
\text { and } 300 \mathrm{~Hz} .\end{array}$ \\
\hline Magnetostriction & $\begin{array}{c}\text { The fundamental frequency is } 100 \mathrm{~Hz}, \text { and the } \\
\text { vibration of the core caused by magnetostriction is } \\
\text { still very strong at the frequency of } 600 \mathrm{~Hz} .\end{array}$ \\
\hline Circuit breaker operation & $\begin{array}{c}\text { The vibration frequency range is } 100 \mathrm{~Hz}-1 \mathrm{kHz} . \\
\text { To ground short circuit }\end{array}$ \\
\hline $\begin{array}{c}\text { The vibration frequency ranges from } 100 \mathrm{~Hz} \text { to } \\
500 \mathrm{~Hz} .\end{array}$ \\
\hline
\end{tabular}

It can be seen from this that the vibration signal can effectively distinguish the mechanical fault of low frequency vibration from the insulation fault of high frequency vibration and the various types of mechanical fault according to its frequency difference.

\section{Mechanical fault detection of GIS}

The research on fault diagnosis can usually be divided into four levels: first, the existence of fault can be determined; second, the location of fault can be determined; third, the damage degree of fault can be determined; and fourth, residual life prediction and reliability assessment [12]. At present, GIS mechanical fault diagnosis is mainly based on the first three levels. The vibration signal extracted from the operating equipment is used to analyse and identify the fault. The improvement of methods mainly lies in the selection of signal feature extraction and analysis methods.

\subsection{Traditional diagnostic methods}

Because the vibration signals generated by normal operation or fault of GIS are obvious different from other signals, and the frequency range of various fault types is quite different, most of the mechanical fault detection is based on the extraction and analysis of vibration signals since the birth of GIS fault detection technology.

\subsubsection{Signal feature extraction}

It is very important for fault diagnosis to extract the effective information from the acquisition signal. The optimization of feature extraction can further improve the accuracy and efficiency of fault diagnosis. At present, the commonly used methods are wavelet transform, fast Fourier transform (FFT), independent component analysis. In [13], a mechanical defect diagnosis 
method was proposed to analyse abnormal vibration of GIS, and a wavelet noise reduction approach was utilized to process the vibration signal to obtain the corresponding spectrum diagram. By analysing the signal components of each frequency segment in the spectrum, the mechanical fault type of GIS is determined. Similarly, in [14], a GIS mechanical defect diagnosis system and method is proposed, based on independent component analysis and denoising. The independent component method is used to achieve ideal signal-to-noise separation for the collected vibration signals, and the corresponding spectrum diagram is obtained. Then it was matched with the GIS mechanical fault frequency characteristics to determine the fault type. In [15], a GIS mechanical condition detection system based on vibration signals is developed, focusing on the mechanical vibration of TV and busbar faults, which uses traditional wavelets analysis and FFT analysis methods to feature extraction. These are all valid experiments with traditional methods.

Empirical Mode Decomposition (EMD) is an novel signal decomposition algorithm, which is adapted to non-linear and non-stationary signals. Once it was proposed, it attracted widespread attention. Compared with traditional analysis methods, EMD overcomes the limitations of the FFT transform, and unlike the wavelet transform, the wavelet basis function must be selected. It has great time-frequency resolution and adaptability, and can highlight small details in the signals. The main processing steps of EMD are shown in figure 3.

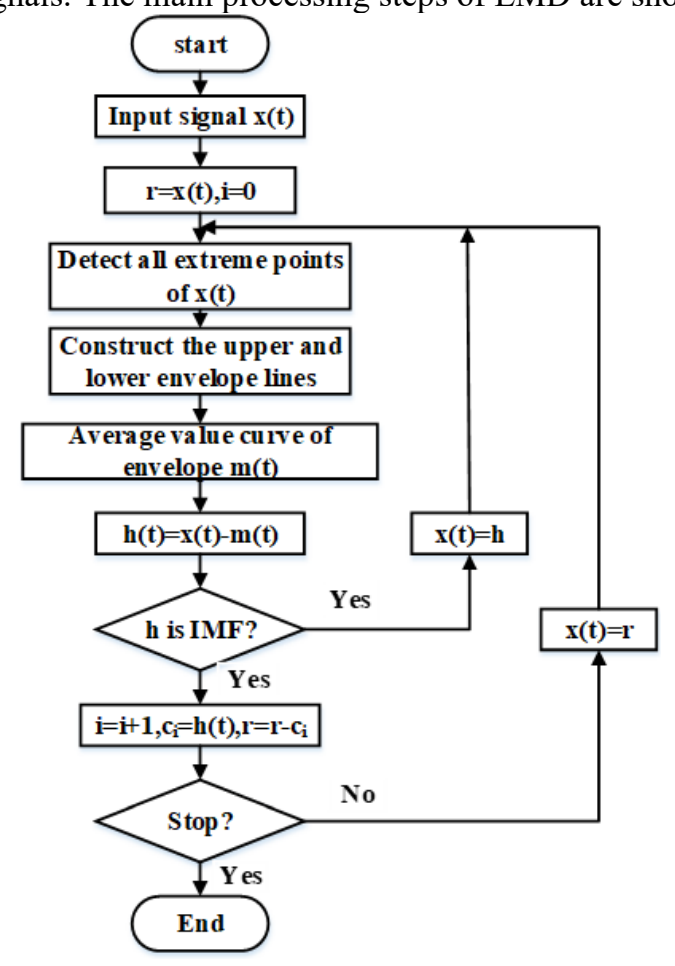

Figure.3. EMD algorithm flow

In [16], empirical mode decomposition is used to extract vibration signals and performed time-frequency analysis; In [17], the researchers apply EMD algorithm to the processing of vibration signals, and the power spectral density function and the vector space model are used to obtain the matrix. The mechanical state of the GIS is monitored by contrasting the power matrix similarity of the vibration signals.

Although empirical mode decomposition is a more mature method of time-frequency signal analysis, there are still modal aliasing phenomena and phenomena that are prone to produce false components. To this problem, in [18], the Ensemble Empirical Mode 
Decomposition(EEMD) is used to extract the characteristic amount of the vibration signal, and then the Hilbert transform of the signal obtained through the EEMD is analysed, verified by the GIS vibration signal detection test platform to obtain a more accurate characteristic criterion.

Variational Modal Decomposition (VMD) is an adaptive, non-recursive signal decomposition algorithm based on EMD. It has obvious advantages over EMD and EEMD in terms of signal decomposition accuracy and noise robustness. In [19], a mechanical fault diagnosis method of GIS is published, depended on chaotic valley-buffering algorithm to optimize the variational mode decomposition (VMD ) parameters. Finally, VMD entropy, root mean square and the principles of minimum Euclidean distance were utilized to identify the feature vector of samples, and the diagnosis of mechanical fault of GIS is realized.

The above is a more common application of feature extraction technology in GIS machinery fault diagnosis. With its continuous improvement, GIS machinery fault diagnosis technology based on vibration signals will have greater development.

\subsubsection{Fault identification method}

\section{1) Traditional analysis method}

After the fault features are extracted, it is crucial to effectively analyze and match the known fault features. Because the vibration frequency generated by GIS mechanical faults has a large difference and the information displayed in the time-domain waveform of the extracted signal shows less information, the most common method currently is to determine the type of fault through spectrum analysis. In [20], a single-fault experimental study on the looseness of GIS bus bars is carried out. After an in-depth understanding of the fault mechanism, a simulation experiment was performed through the GIS mechanical vibration detection test platform. The fault characteristics were analysed by spectrum. The results show that in addition to the obvious characteristics of the vibration signal at the frequency of 100 $\mathrm{Hz}$, the vibration signal also changes in the frequency range of 1000 to $2000 \mathrm{~Hz}$, especially at the frequency of $1400 \mathrm{~Hz}$. In [13] and [14], after processing the signals, the types of GIS mechanical faults were also determined by analysing the signal components in each frequency band of the spectrogram. In [21], dynamic time warping algorithm (DTW) to perform time-frequency signals is used. Other researchers judged the ratio of vibration amplitudes at different frequencies as characteristic values. After the frequency analysis of the vibration signal in [10], the amplitude ratio between different frequencies is used as the criterion for judging the contact state of the GIS isolating switch. In the same method, the problem of bus looseness is explored in [22].

Compared with spectrum analysis, power spectrum can represent the relationship between signal power and frequency, and its analysis is more effective. In [23], spectrum analysis and power spectrum analysis and diagnosis of GIS vibration signals is carried out at the same time, and then obtained fingerprints through census for comparison to effectively diagnose the fault location. It is confirmed that power spectrum analysis can reflect abnormal states better than spectrum.

Analysing the type of fault by just the frequency spectrum may miss information hard to detect. In [5], the Hilbert-Huang transform is applied to the processing of GIS vibration signals. By comparing the Hilbert spectra of vibration signals with normal operation of GIS and suspected faults, the changes of the amplitude of each frequency component with time were obtained. Then the marginal spectrum of the normal signal and the abnormal signal is compared to get the fault feature quantity of the GIS equipment, so as to determine whether it is faulty. This method has some effects on fault identification, but the fault characteristics displayed by it are not accurate and require a large amount of measured data. So there is space 
for improvement of this method. Based on this, in [18], EEMD is combined with Hilbert transform to obtain more accurate fault characteristics and improve fault diagnosis efficiency. 2) Fault diagnosis method based on feature classification

As a kind of complex structure equipment, GIS not only contains a lot of electrical devices, but also a large number of mechanical devices. GIS has high reliability, so it is difficult to obtain its fault data, and it often has fewer measured samples. As mentioned above, most methods of GIS mechanical fault diagnosis are verified on experimental test platforms. Because GIS fault detection lacks experience accumulation, algorithms that rely on subjective experience cannot be applied, such as fuzzy theory. The stability of genetic algorithms is poor. The artificial neural network relies heavily on data and is not applicable to small sample problems. However, as a classifier, support vector machine (SVM) has a relatively small empirical risk and confidence range, and can achieve such small sample classification. In the past two years, this method has begun to be applied to GIS mechanical failure. In [24], the combination of wavelet packet decomposition theory and vector machine theory was used to extract fault features and identify the state of high-voltage circuit breakers. Aiming at the problem of high-voltage circuit breaker fault vibration signals caused by insufficient mechanical lubrication, it is difficult to determine the fault if the energy entropy is directly used to extract energy entropy for feature extraction. An energy segmentation method is proposed in [25], such as EMD, to extract feature quantities and combined with support vector machines. Then a method is proposed for diagnosing mechanical faults of high-voltage circuit breakers, verified with common faults. In [26], the amplitude ratios of vibrations at different frequencies are also used as eigenvalues, and then SVM is used for classification and recognition. In [11], VMD energy entropy and root mean square value as the feature values is used, and support vector machine is used, which is combined with kernel principal component analysis to diagnose. The diagnosis success rate was high.

Aiming at the limitation of SVM in the classification of complex faults, the researchers put forward a failure diagnosis method based on the combination of frequency feature extraction of coherent functions and multi-layer classifiers, which combined two one-class support vector machines (OCSVMS) and one support vector machine (SVM) into a multilayer classifier. The first OCSVM classifier was utilized to differentiate between normal and abnormal conditions in actual detection. The second OCSVM classifier was utilized to distinguish between known and unknown faults, and solve the problem of error diagnosis of unknown faults. The fault type is determined by support vector machines in failure recognition [27]. This method makes the organic combination of failure judgment, type identification, and condition monitoring to maximize the advantages of SVM.

In addition, there are some classification algorithms that have been used more, such as the principle of cluster analysis. In [28], the neighbourhood algorithm is combined with the fuzzy C-mean algorithm (FCM) to obtain the final classification result to calculate the initial cluster center. Not only can it solve the problem of storage requirements in the neighbourhood algorithm, it has a large amount of calculation and a long calculation time, and it also overcomes the inaccuracy of the cluster center obtained from FCM, which causes the low accuracy of GIS mechanical fault diagnosis.

\subsection{Other diagnostic methods}

In recent years, with the researchers' emphasis on GIS mechanical failure and the rapid development of GIS online monitoring technology, the judgment of GIS mechanical failure depends not only on vibration signals. Some researchers have used noise signals to judge and locate GIS faults, or comprehensively analyse sound and vibration signals to determine the type of mechanical faults. A self-developed set of front-end abnormal sound online acquisition equipment is introduced in [29], and a signal fault diagnosis algorithm based on 
an online sequence over-limit learning machine is proposed. The accuracy of the algorithm increases with the increase of the number of GIS sound waves. The device can make up for the blank problems in the field of signal acquisition and online monitoring of early failures of GIS based on acoustic signal analysis. The acoustic characteristics of GIS mechanical faults is summarized in [30], where the time domain, frequency domain and inverse frequency domain laws of different faults are analysed, and a software is designed.

In the future, the means and methods for GIS fault signal collection will become more and more abundant, and the problem of difficult GIS fault signal collection will also be solved.

\section{EARLY FAULT PREDICTION AND RELIABILITY EVALUATION OF GIS}

The above three are the first three levels of fault diagnosis. The first three levels of fault diagnosis are based on the failure of the equipment, and the fault monitoring and maintenance are performed in time. The purpose of fault prediction is to predict the future health status of the device and the time interval from the current status to the fault status by the prediction method, and to extend the life cycle of the device [31]. Especially for GIS, a highly integrated device with complex internal structure, a series of chain reactions may occur. Once GIS is damaged, it will cause a large range of impacts, make maintenance difficult and take a long time. Faults are divided into complete faults and faint faults according to their development degree. Complete faults refer to equipment failures that have developed to the middle and late stages, and the fault characteristics are obvious. Failure to take measures will cause major accidents; Faint faults usually refer to faint faults or potential faults at early stages or the characteristics of the faults are masked and not obvious. Early prevention of weak faults can avoid the occurrence of complete faults, and mechanical fault diagnosis has gradually shifted from complete fault maintenance to weak fault state evaluation and prediction [12]. A GIS mechanical failure approach method based on KL divergence is put forward in [32]. The kernel density estimation method was used to estimate the probability density of the original measurement signal. The degree of fault occurrence was judged by calculating the divergence between the normal signal and the fault signal. This method can discover potential faults in GIS and locate faults at an early stage.

As a kind of complicated equipment, GIS may cause other faults if one kind of fault occurs. The symptoms it shows are ambiguous and the system needs to sort out the relationship between their failures. The GIS fault tree is established in [3], and the logical relationship between GIS faults is sorted out. Then the reliability of GIS is evaluated. A more in-depth analysis and research on the causes of GIS failures has shown that the failure of manufacturing quality and the lack of strict control over the installation are the main reasons for GIS failures. In [33], Petri net theory is organically combined with fuzzy inference rules, and fuzzy Petri net (FPN) is proposed, which has fast and accurate parallel reasoning capabilities. It uses historical fault data and FPN forward matrix reasoning to perform reliability analysis on GIS. In the case of a known fault phenomenon, the cause of the fault is found out by the reverse fuzzy reasoning rule. Compared with the fault tree, this method has faster and more accurate reliability analysis and fault diagnosis, but it requires a large number of statistical fault cases in order to establish a more comprehensive fault diagnosis model. In [34], according to the actual functional requirements of GIS condition monitoring, a GIS intelligent monitoring status information flow modeling method in accordance with the IEC 61850 communication standard was proposed, and a GIS condition monitoring IED model was established. And the architecture and communication system of GIS intelligent monitoring system are given. In the future, substations will become more intelligent, and data sharing will become more and more convenient. Intelligent diagnosis systems that integrate 
monitoring and early warning, intelligent diagnosis, and condition assessment will become an inevitable trend.

At present, it is still difficult to obtain the entire process data of the state change of GIS mechanical failure, which requires us to make a prejudgment of the monitoring status based on the effective learning of normal operation data.

\section{SUMMARY AND PROSPECT}

In recent years, as one of the main failures of GIS, mechanical failure has attracted more and more attention from researchers. The principle of GIS mechanical failure and its detection technology have been further developed. This paper systematically summarizes the research status of GIS mechanical fault diagnosis technology at home and abroad, and summarizes the characteristics of GIS mechanical faults, fault judgment and location technology, early fault prediction and reliability evaluation of GIS.

1) The research of GIS mechanical faults mostly focuses on single faults, and there are few researches on the types of faults that coexist with multiple mechanical faults. This requires in-depth research on multi-feature signal extraction and processing techniques. Moreover, the verification of diagnostic methods is mostly performed on a simulation test platform, and the effect of actual situation verification is difficult to guarantee.

2) Due to the difficulty of GIS maintenance and the large impact of faults, the research on GIS condition assessment is very necessary, but there are few studies in this area.

3) There is no systematic establishment of a full database of time-frequency maps and acoustic characteristics of various types of GIS faults, which is not conducive to ensuring the accuracy and efficiency of GIS mechanical fault diagnosis.

The above is a summary of the problems at this stage. I hope this article can provide directions for future research on GIS mechanical fault diagnosis.

\section{ACKNOWLEDGEMENT}

This work was supported by the Subsidized Project for Postgraduates' Innovative Fund in Scientific Research of Huaqiao of University.

\section{REFERENCES}

1. Wang Changchang, Li Fuqi, Gao Sheng you. On-line Monitoring and Diagnosis for Power Equipment[M]. Beijing: Tsinghua University Press, 2006:252-254.

2. Zuo Yafang.Operation maintenance and troubleshooting of GIS. Beijing: China Electric Power Press, 2013: 12-15.

3. Cui Yangliu, Ma Hongzhong, Wang Taoyun,et al. Fault Analysis of GIS Based on Fault Tree Theory[J]. High Voltage Apparatus,2015(7):125-129.

4. Sun Q, Cao T, Hou Y, et al. Detection and Analysis Based on the Abnormal Mechanical Vibration Signal of GIS[C]. FifthInternational Conference on Instrumentation and Measurement Computer, Communication and Control (IMCCC). Qinhuangdao,

China: IEEE, 2016.

5. Xu Tianle, Ma Hongzhong, Chen Kai, et al. Diagnosis of GIS Failure Based on HHT Method for Vibration Signals[J]. Electric Power,2013, 46(3):39-42.

6. Chen Xuanhong, Gaoyang, Xu Aolan. Mechanical failure principle of GIS equipment [J]. Shandong Industrial Technology, 2017 (19): 162-162. 
7. Li Kai, Xu Honghua, Chen Bingbing,et al.Research on vibration mechanism and natural frequency in GIS[J]. Electrical Measurement and Instrumentation, 2017, 54(3),14-18.

8. Yang Jinggang, Liu Yuan, Song Siqi, et al.Research on Vibration Detection Technology of GIS Mechanical Defects[J].High Voltage Apparatus,2018, 54(11):94-98.

9. Qi Weidong, Niubo, Hu Degui, et al. Vibration Simulation of GIS Horizontal Bus Shell Based on FEM[J]. High Voltage Apparatus,2018,54(6):46-59.

10. Jia Yongyong, Wu Bang, Shan'yuan Sun, et al. Research of the vibration characteristics in GIS Disconnector under different Contact State[C]. 2018 IEEE International Conference on High Voltage Engineering and Application (ICHVE), ATHENS, Greece,2018.

11. He Siyang, Zhang Tangsong, Zhou Lan,et al.GIS fault diagnosis strategy based on feature classification algorithm[J].Automation and Instruments,2019, 232(02):203-206.

12. Wang Guobiao, He Zhengjia, Chen Xuefeng, et al.Basic Research on Machinery Fault Diagnosis-What is the Prescription[J].Journal of Mechanical Engineering,2013, 49(1):63-72.

13. Sun Qingsheng ; Cao Tao,et al.Detection and Analysis Based on the Abnormal Mechanical Vibration Signal of GIS[C].Proceedings of 5th International Conference on Instrumentation and Measurement, Computer, Communication, and Control, Qinhuangdao, China, 2015.

14. State Grid Corporation. GIS Machinery Defect Diagnosis System and Method Based on Independent Component Analysis Denoising: China, 201510971975.3 [P]. 2016-06-01.

15. Huang Jinjian,Yang Baokun.GIS Mechanical Condition Detection System Based on Vibration Signaland Its Application[J].Guangxi Electric Power, 2015, 38(5):55-59.

16. Sun Qingsheng, Cao Tao, Zhou Zhangbin, et al. Mechanical Vibration Signal Analysis of GIS Based on EMD Algorithms [J]. Electrical Applications, 2016 (20): 79-84.

17. Zhigang Wu, Bing Liu, Lijuan Wu, et al. Analysis of GIS Mechanical Vibration Signal Based on EMD Algorithm[C]. 3rd International Conference on Information Science and Control Engineering (ICISCE),Beijing, China: IEEE , 2016.

18. Zheng Hao, Zhu Shenglong, Ouyang Yu, et al. Time - frequency Analysis of GIS Mechanical Vibration Signals Based on Improved HHT Algorithm[J]. Computer Technology and Applications,2018,40(1):15-18.

19. Gao Xiaodong, Lv Shouguo, Zhuang Yanfei, et al. Method for Diagnosis of Gas Insulated Switchgear Based on VMD Parameters Optimized by Chaotic Cuckoo Algorithm[J]. 13th IEEE Conference on Industrial Electronics and Applications (ICIEA), Wuhan, China,2018.

20. Huang Qing, Wei Xu, Xu Jiangang, et al.Research of GIS Bus Loose Contact Fault Diagnosis Techniques Based on Mechanical Vibration[J].High Voltage Apparatus, 2017,53(11):99-108.

21. Michel Landry, FranÇois Leonard, Champlain Landry, et al. An Improved Vibration Analysis Algorithm as a Diagnostic Tool for Detecting Mechanical Anomalies on Power Circuit Breakers[J]. IEEE Transactions on Power Delivery, 2008, 23(4):1986 - 1994.

22. Liu Yuan, Jia Yongyong, Yang Jinggang. Research of Mechanical State Diagnosis Techniques in GIS Bus Connector Based on Mechanical Vibration[C]. 12th International Conference on the Properties and Applications of Dielectric Materials (ICPADM), Xi'an, China,2018. 
23. Cheng Lin.Research on UHV GIS/HGIS Vibration Detection and Diagnosis Method[J].Electric Power Construction,2009,30 (7):17-19.

24. Sun Laijun.A New Method of Fault Diagnosis for High Voltage Circuit Breakers Based on Vibration Signals[J].Proceedings of the CSEE, 2006, 26(6): 157-161.

25. Huang Jian, Hu Xiaoguang, Gong Yunan.Machinery Fault Diagnosis of High Voltage Circuit Breaker Based on Empirical Mode Decomposition[J].Proceedings of the CSEE,2011, 31(12): 108-113.

26. Feng Ying, Lan Jian, et al. Bolt looseness fault diagnosis of GIS based on vibration signal amplitude[C]. International Conference on Mechanical, Electric and Industrial Engineering, Hangzhou, China,2018.

27. Yuan Yang, Ma Suliang, Wu Jianwen, et al. Frequency Feature Learning from Vibration Information of GIS for Mechanical Fault Detection[J]. Sensors, 2019, 19(8): 1949.

28. Shen X, Lin Z, Peng G, et al. Research on mechanical fault diagnosis of Ultra high voltage GIS based on the combination of neighbour algorithm and FCM[C]. IEEE PES Asia-Pacific Power and Energy Engineering Conference (APPEEC), Xi'an, China, 2016.

29. Wang Zhe, Gao Wei, Yang Fan, Leiyuan. An On-line abnormal sound monitoring device for the diagnosis and location of mechanical defects in GIS ontology[J]. Electronic measurement technology, 41 (21): 103-108.

30. Shang Yu,Liu Qiang ,Niu Bo ,et al. Mechanical Fault Diagnosis System Based on Acoustic Feature Analysis in Gas Insulated Switchgear[C]. 1st International Conference on Electrical Materials and Power Equipment (ICEMPE), Xi'an, China,

31. Xu Yuanjun, Pan Hongxia.Study on Tendency Prediction in Equipment Failure[J]. Journal of Vibration,Measurement and Diagnosis, 2006, 26(4): 305-308.

32. Hou Yan,Zhao Tong,Hao Zheng,et al.Discriminant Approach to the GIS Mechanical Fault Diagnosis Based on the K-L Divergence of Vibration Signals[C].IEEE International Conference on High Voltage Engineering and Application (ICHVE), Chengdu, China,2016.

33. Wang Taoyun, Ma Hongzhong, Cui Yangliu, et al. Fault diagnosis and reliability analysis for GIS based on fuzzy Petri net[J].Advanced Technology of Electrical Engineering and Energy, 2016, 35(5):67-73.

34. Du Fengqing, Sheng Gehao, Xu Jian, et al. Information modeling for GIS smart monitoring based on IEC61850 and development of information interaction system[J]. Electric Power Automation Equipment, 2013, 33(6): 163-167. 